\title{
A comparative investigation on strain induced crystallization for graphene and carbon nanotubes filled natural rubber composites
}

\author{
D. H. Fu, Y. H. Zhan, N. Yan, H. S. Xia*
}

State Key Laboratory of Polymer Materials Engineering, Polymer Research Institute, Sichuan University, 610065

Chengdu, China

Received 18 November 2014; accepted in revised form 26 January 2015

\begin{abstract}
Natural rubber containing graphene and carbon nanotubes (CNTs) composites were prepared by ultrasonicallyassisted latex mixing. Natural rubber filled by both graphene and CNTs show significant enhanced tensile strength, while graphene exhibits a better reinforcing effect than CNTs. Strain-induced crystallization in natural rubber composites during stretching was determined by synchrotron wide-angle X-ray diffraction. With the addition of CNTs or graphene, the crystallization for natural rubber occurs at a lower strain compared to unfilled natural rubber, and the strain amplification effects were observed. The incorporation of graphene results in a faster strain-induced crystallization rate and a higher crystallinity compared to CNTs. The entanglement-bound rubber tube model was used to analyze the chain network structure and determine the network parameters of composites. The results show that the addition of graphene or CNTs has an influence on the molecular network structure and improves the contribution of entanglement to the conformational constraint, while graphene has a more marked effect than CNTs.
\end{abstract}

Keywords: rubber, strain-induced crystallization, tube model, graphene, carbon nanotube

\section{Introduction}

Natural rubber (NR) is used extensively in a variety of applications, due to its outstanding elastic property and good cracking resistance, arising from its ability to crystallize upon stretching [1-4]. The addition of nanoparticles offers the opportunity to render versatility in NR and extend its applications that unlikely to achieve by using NR alone. Graphene (GE) and carbon nanotubes (CNTs) are among the most fascinating nanoparticles, which have received numerous studies because of their intrinsic excellent properties [5, 6], such as high modulus [7], tensile strength [8], thermal $[9,10]$ and electrical conductivity $[11,12]$. GE has been used to prepare NR nanocomposites by an ultrasonically assisted latex mixing and in-situ reduction process [13-15], the mechanical properties were significantly improved at a low content of GE [14]. The reinforcement effect of graphene is believed to be related to its effect on strain induced crystallization (SIC).

Synchrotron X-ray diffraction (WAXD) allows the in-situ examination of SIC in NR composites during stretching [13, 16-20]. Results from extensive studies demonstrated the preferred chain orientations and prominent enhancement of SIC upon elongation in the filled rubber composites. Song et al. [19] investigated the SIC of natural rubber containing various amounts of CNTs and carbon black (CB) by synchrotron X-ray scattering. It was found that the rubber containing CNTs shows early onset point of SIC and CNTs helps to orient the chains and SIC during the uniaxial stretching. However, the CB in rubber

\footnotetext{
*Corresponding author, e-mail: xiahs@scu.edu.cn

(C) BME-PT
} 
act to interrupt the SIC. Ozbas et al. [20] compared the effect of graphene and CB on the SIC by synchrotron X-ray scattering. The results showed that the onset of crystallization occurs at significantly lower strains for GE-filled NR samples compared with CB-filled NR Neat-NR exhibits SIC around a strain of 2.25, while incorporation of 1 and $4 \mathrm{wt} \% \mathrm{GE}$ shifts the crystallization to strains of 1.25 and 0.75 , respectively. In contrast, loadings of $16 \mathrm{wt} \% \mathrm{CB}$ do not significantly shift the critical strain for crystallization. Despite many studies conducted on the SIC of NR, a comparative study on the influences of GE and CNTs on the SIC and rubber network parameters is still missing.

The established molecular statistical theories of crosslinked and entangled polymers assume that the restricted phase space (due to the entanglements) available to the chains only affects crosslink fluctuations. Nevertheless, these theories give no insight into the relationship between the configurational constraint of entire network chain and stress-strain behavior of crosslinked network. Edwards [21, 22] developed the concept of the tube model by conceiving that the entanglements act along the entire contour length of network chain segments. The introduction of tube model not only allows to reveal non-isotropic crosslink fluctuations to all chain segments of the network via constraining virtual tubes around the network chains, but also enables proper crosslink separation and constraint contribution to stress-strain behavior. Although the tube model has reliability in investigating stress-strain behavior to determine the network parameters of the unfilled rubber, it is not applicable in depicting the filled rubber network because of its complexity [23]. Funt [24] first proposed an entanglement bound rubber tube (EBT) model to describe the contribution of entanglement, which extends tube model to the filled system. Thereafter, EBT model has been used to describe the effect of carbon black [25] and clay [26] on the mechanical behavior of rubber. However, few works concerned on the influence of GE and CNTs on the NR network structure through the EBT model.

In this study, the influences of GE and CNTs on the strain-induced crystallization were investigated by synchrotron WAXD measurements. EBT model was used in combination with stress-strain experiments to calculate the network parameters, and then to assess the contribution of entanglement to the mechanical property. The results provide new insight into the discrepancy of the reinforcing effect between GE and CNTs.

\section{Experimental \\ 2.1. Materials}

The commercial used natural rubber (NR) in this study was NR (SCR 5) from Yunnan Natural Rubber Industry Co., Ltd, Kunming, China. NR latex (NR content: $60 \mathrm{wt} \%$ ) was purchased from Chengdu Fangzheng Co., Ltd (China). Flake Graphite with an average thickness of $\sim 75 \mu \mathrm{m}$ was obtained from Qingdao Dahe Graphite Co., Ltd (China). Hydroxyl multi-walled carbon nanotubes (CNTs, $\sim 3 \mathrm{wt} \%$ ) with a diameter of 10 20 nm were obtained from Chengdu Institute of Organic Chemistry, Chinese Academy of Sciences. Formic acid was purchased from Tianjin Bodi Chemical Reagent Co., Ltd (China). Other reagents were obtained from Chendu Kelong Chemical Reagent Company (China).

\subsection{Preparation of the NR composites}

NR composites containing $1.76 \mathrm{wt} \%$ CNTs or GE were prepared through the previous route called ultrasonically assisted latex mixing and in-situ reduction process $[14,15]$. Firstly, natural flake graphite was oxidized to graphene oxide (GO) according to the Hummer's method [27]. Then, $2.5 \mathrm{mg} / \mathrm{mL}$ GO aqueous solution was prepared by bath sonication (KQ-250DE, $40 \mathrm{kHz}$, Kunshan Ultrasonic Instrument Co., Ltd, China) at $40^{\circ} \mathrm{C}$ for $1 \mathrm{~h}$. Then an appropriate quantity (16.6 g) of NR latex was sequentially dispersed into the GO solution by sonication for $1 \mathrm{~h}$. After the coagulation and drying process, the NR/GO latex mixture was obtained. Then hydrazine hydrate was added into the GO/NR latex and the mixture was subjected to sonication. After the coagulation and drying process, the NR/GE masterbatch was obtained. Vulcanized NR samples were prepared in an open twin roll mill at room temperature according to the recipes outlined in Table 1 . The resultant compounds were compression molded into sheets at a curing temperature of $150^{\circ} \mathrm{C}$ and a pressure of $10 \mathrm{MPa}$ for $5 \mathrm{~min}$, and then cooled for 3 min under pressure at room temperature.

For a comparative purpose, the natural rubber/carbon nanotubes (CNTs/NR) composites with $1.76 \mathrm{wt} \%$ filler content were also prepared by a latex mixing route. 
Table 1. Recipes for preparing NR and NR composites containing $1.76 \mathrm{wt} \% \mathrm{CNTs}$ or GE

\begin{tabular}{|l|c|c|c|}
\hline \multicolumn{1}{|c|}{ Samples } & NR & CNTs/NR & GE/NR \\
\hline Raw NR & 80 & 80 & 80 \\
\hline $\begin{array}{l}\text { NR masterbatch containing } \\
10 \mathrm{wt} \% \text { GE or CNT }\end{array}$ & 0 & 20 & 20 \\
\hline Zinc oxide & 5 & 5 & 5 \\
\hline Stearic acid & 2 & 2 & 2 \\
\hline Sulfur & 3 & 3 & 3 \\
\hline Antioxidant $(4010 \mathrm{NA})$ & 2 & 2 & 2 \\
\hline Accelerator $\left(\mathrm{CBS}^{\mathrm{a}}\right)$ & 1.5 & 1.5 & 1.5 \\
\hline Accelerator $\left(\mathrm{MBT}^{\mathrm{b}}\right)$ & 0.1 & 0.1 & 0.1 \\
\hline
\end{tabular}

${ }^{a} \mathrm{CBS}, \mathrm{N}$-cyclohexyl-2-benzothiazole-sulfenamide

${ }^{\mathrm{b}} \mathrm{MBT}$, 2-mercaptobenzothiazole

\subsection{Characterization}

Synchrotron wide-angle X-ray diffraction (WAXD) measurements were carried out at room temperature using a U7B beam-line in the National Synchrotron Radiation Laboratory, Hefei, China. The wavelength was $0.154 \mathrm{~nm}$. The specimen was symmetrically stretched at a strain rate of $10 \mathrm{~mm} / \mathrm{min}$ to the predetermined elongation, allowing the X-ray to irradiate almost the same specimen position. The exposure time for each image was $180 \mathrm{~s}$. The two-dimensional (2D) WAXD patterns were recorded by a Mar CCD 165 X-ray detector system and analyzed by the Fit2D software package.

The tensile strength was tested using an Instron (5567) universal test machine at room temperature. The deformation rate was $500 \mathrm{~mm} / \mathrm{min}$. The initial width and thickness of those samples were 4 and $2 \mathrm{~mm}$, respectively. All tests were carried out on a minimum of five samples and the reported results are average values.

The swelling behavior was tested as follows: the cured samples were cut into strips with dimensions of $30 \mathrm{~mm} \times 5 \mathrm{~mm} \times 2 \mathrm{~mm}$, and then immersed in toluene at $25^{\circ} \mathrm{C}$ for $48 \mathrm{~h}$ to reach their equilibrium swelling. Afterward, the samples were dried at $60^{\circ} \mathrm{C}$ until constant weights were obtained. The toluene uptake per gram of rubber $(Q)$ was determined using Equation (1):

$Q=\frac{w_{\mathrm{s}}-w_{\mathrm{d}}}{w_{\mathrm{o}} \cdot \varphi_{\text {rubber }}}$

where $w_{\mathrm{s}}$ is the swollen weight, $w_{\mathrm{d}}$ is the dried weight, $w_{\mathrm{o}}$ is the original weight and $\varphi_{\text {rubber }}$ is the mass fraction of the rubber in the composites. The filler-rubber interaction in the composite was evaluated by the $Q_{\mathrm{f}} / Q_{\mathrm{g}}\left(Q_{\mathrm{f}}\right.$ and $Q_{\mathrm{g}}$ are the toluene uptakes per gram for filled and neat rubber respectively) [28]. The lower $Q_{\mathrm{f}} / Q_{\mathrm{g}}$ value means the higher interaction extent between the filler and matrix [29].

The bound rubber content was tested based on the method reported by Leblanc and Hardy [30]. Uncured sample $(0.5 \mathrm{~g})$ was cut into small pieces and placed into a steel wire net $\left(m_{1}\right)$. The net was closed and weighed $\left(m_{2}\right)$, and then immersed in toluene at room temperature for $72 \mathrm{~h}$ during which the sample was washed with fresh solvent every $24 \mathrm{~h}$. The net was slowly removed from the solvent and dried at $40^{\circ} \mathrm{C}$ under vacuum for a few hours until a constant weight $\left(m_{3}\right)$ was achieved. The amount of bound rubber (as the weight percent, $\mathrm{wt} \%$ ) of the initial rubber content of the uncured sample was given by Equation (2):

$B d R[\%]=\frac{m_{0}-\left(m_{2}-m_{3}\right)}{m_{0}} \cdot 100$

where $m_{0}$ is the rubber content in the sample given by $m_{0}=\left(m_{2}-m_{1}\right) \cdot \varphi_{\text {rubber }}\left(m_{1}\right.$ is the mass of the empty net and $\varphi_{\text {rubber }}$ is the mass fraction of the rubber in the composites.), $m_{2}$ is the mass of the net plus the unextracted sample, and $m_{3}$ is the mass of the basket plus the extracted dried sample.

\section{Results and discussion}

\subsection{Stress-strain behavior}

The stress-strain curves for the unfilled NR and NR nanocomposites with GE or CNTs are shown in Figure 1. The NR exhibits marked increase in tensile strength with low contents of GE or CNTs. The tensile strength was increased by $98.4 \%$ from $12.4 \mathrm{MPa}$ for unfilled NR to 24.6 MPa for the nanocomposite with $1.76 \mathrm{wt} \%$ GE. The NR/CNTs containing $1.76 \mathrm{wt} \%$ of CNTs showed a tensile strength of 18.6 $\mathrm{MPa}$. It is clear that GE shows an evidently

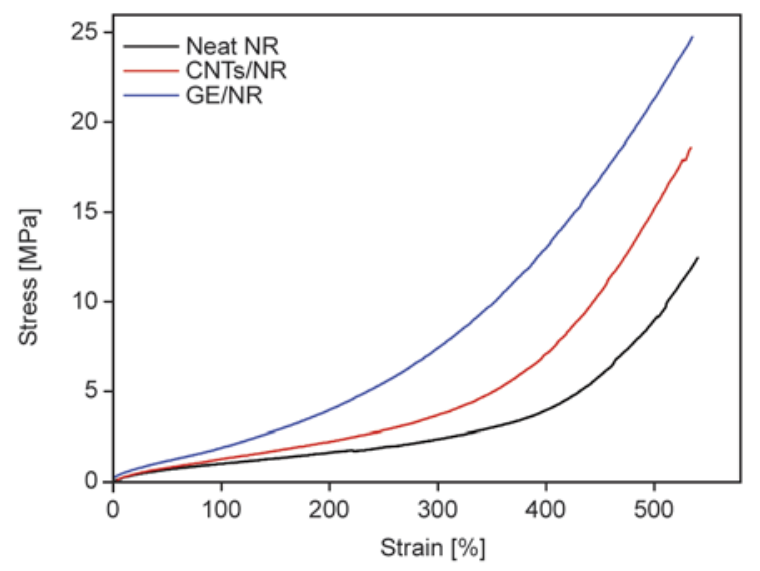

Figure 1. The stress-strain curves of filled and unfilled NR 
better reinforcing effect on NR than CNTs. Additionally, both GE and CNTs at low contents exhibit the comparable reinforcing effect to the conventional filler at high loading, indicating the reinforcing advantages of nanoparticles.

In order to understand the reinforcing effect of GE and CNTs, we compared the crosslink density, bound rubber content and filler-rubber interaction of the rubber nanocomposites. The crosslink chains density $\left(v_{\mathrm{e}}\right)$ can be obtained according to Equation (3) [31]:

$\sigma=v_{\mathrm{e}} k T\left(\alpha-\alpha^{-2}\right)$

where $\sigma$ is the force per unit area, $v_{\mathrm{e}}$ is the crosslink chains density, $k$ is the Boltzmann constant, $T$ is the absolute temperature, and $\alpha$ is the elongation ratio ( $\alpha=l / l_{0}$, in which $l_{0}$ is the initial length of the sample and $l$ is that of the elongated sample). The bound rubber content was tested based on the method reported by Leblanc and Hardy [30] (Equation (2)). The filler-rubber interaction in the composites was evaluated by $Q_{\mathrm{f}} / Q_{\mathrm{g}}$. The crosslink density, bound rubber content and filler-rubber interaction are listed in Table 2. The crosslink chains density for GE/NR is more than twice higher than that of NR, also much higher than that of CNTs/NR. In addition, the GE/NR composite has a very high bound rubber content, nearly three times higher than the CNTs/NR composite, which suggests stronger interaction between GE and NR. The lower $Q_{\mathrm{f}} / Q_{\mathrm{g}}$ value of NR/GE also indicates a stronger interaction between GE and NR matrix. From those data on the crosslink density, bound rubber content and filler-rubber interaction of the rubber nanocomposites, it can be understood why graphene has a much better reinforcing effect on natural rubber. In order to get deeper understanding into the reinforcing mechanism, further investigations were conducted experimentally by Synchrotron Wide-angle X-ray Diffraction and theoretically through the entanglement-bound rubber tube model.

Table 2. The crosslink density, bound rubber content and filler-rubber interaction for the filled and unfilled NR

\begin{tabular}{|l|c|l|c|}
\hline \multicolumn{1}{|c|}{ Sample } & NR & CNTs/NR & GE/NR \\
\hline$v_{\mathrm{e}} \cdot 10^{4}\left[\mathrm{~mol} / \mathrm{cm}^{3}\right]$ & $0.79 \pm 0.01$ & $0.92 \pm 0.01$ & $1.72 \pm 0.03$ \\
\hline$B d R[\%]$ & 0 & $11.4 \pm 0.2$ & $30.3 \pm 0.4$ \\
\hline$Q_{\mathrm{f}} / Q_{\mathrm{g}}$ & - & $0.89 \pm 0.01$ & $0.85 \pm 0.01$ \\
\hline
\end{tabular}

\subsection{Strain induced crystallization during stretching}

It has long been recognized that the excellent tensile properties of NR originate from its strain-induced crystallization ability. It is of great significance to explore the relation between nanoparticles and strain induced crystallization. SIC was studied via in-situ synchrotron wide-angle X-ray diffraction. The 2D WAXD patterns for unfilled NR and NR containing GE or CNTs during stretching are shown in Figure 2. All images were normalized with respect to the sample thickness change, sample absorption, and beam fluctuation. The intensities of these reflections increase with strain during stretching. NR/GE composite has stronger diffraction intensity compared to unfilled NR and NR/CNTs at the same strain. The evolution of strain-induced crystallization can be identified by the linear diffraction profiles taken along the equator from the 2D WAXD patterns collected at different strains during stretching (Figure 3). The resultant peaks on the equatorial diffraction profiles were fitted with a Gaussian function (Equation (4)):

$I(x)=h \cdot \frac{\exp \left[-\left(x-x_{\mathrm{c}}\right)^{2}\right]}{2 w^{2}}$

where $I(x)$ is the intensity at position $x, x_{\mathrm{c}}$ is the position at the scattering maximum, and $h$ and $w$ are the parameters related to the peak height and peak width, respectively. It can be seen that two distinct diffraction peaks located at $2 \theta \sim 15.3^{\circ}$ corresponding to the 200 plane and $2 \theta \sim 19^{\circ}$ corresponding to the 120 plane increase with strain, and the crystallization occurred at a lower strain for GE/NR compared to that for unfilled NR and CNTs/NR composites. The equatorial reflection peak intensities of $I_{\mathrm{eq}}(200)$ and $I_{\text {eq }}(120)$ were used to assess the crystallinity via quantitative evaluation in mass fractions of crystal and amorphous phase. The crystallinity calculation from the resultant Gaussian peak is described as Equation (5) [32]:

$X_{\mathrm{c}}=\frac{A_{\mathrm{c}}}{A_{\mathrm{c}}-A_{\mathrm{a}}}$

where $A_{\mathrm{c}}$ and $A_{\mathrm{a}}$ represent the integrated intensities of the crystalline and amorphous part, respectively. Figure $3 \mathrm{~b}$ shows the variation of crystallinity with strain for unfilled NR, GE/NR and CNTs/NR com- 

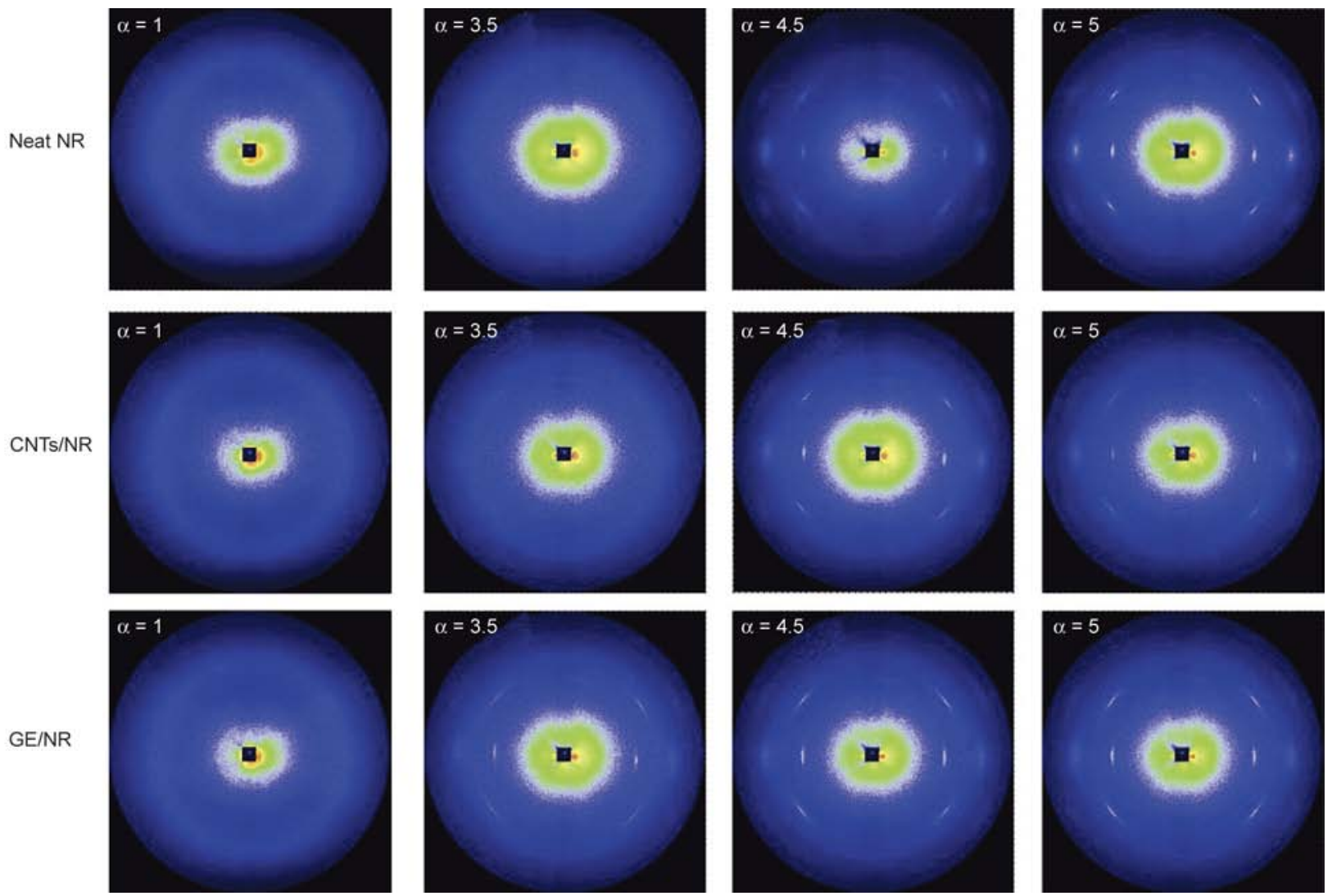

Figure 2. Sequential changes of WAXD patterns of neat NR, CNTs/NR and GE/NR

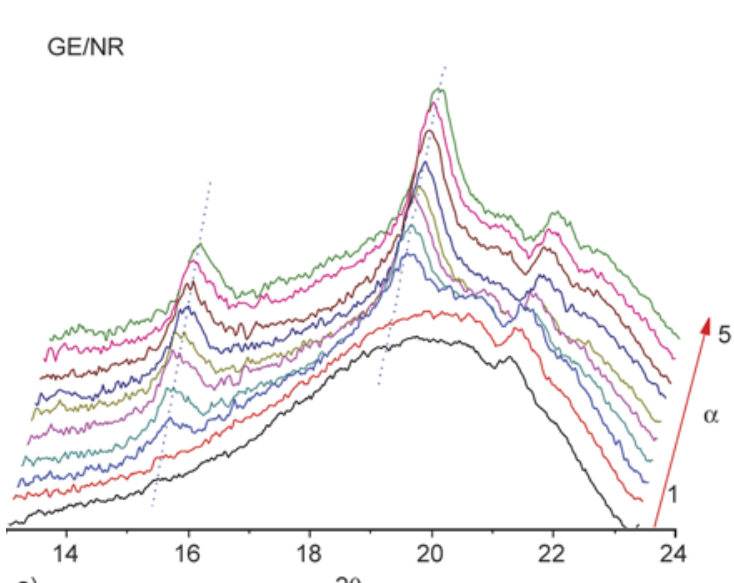

a)

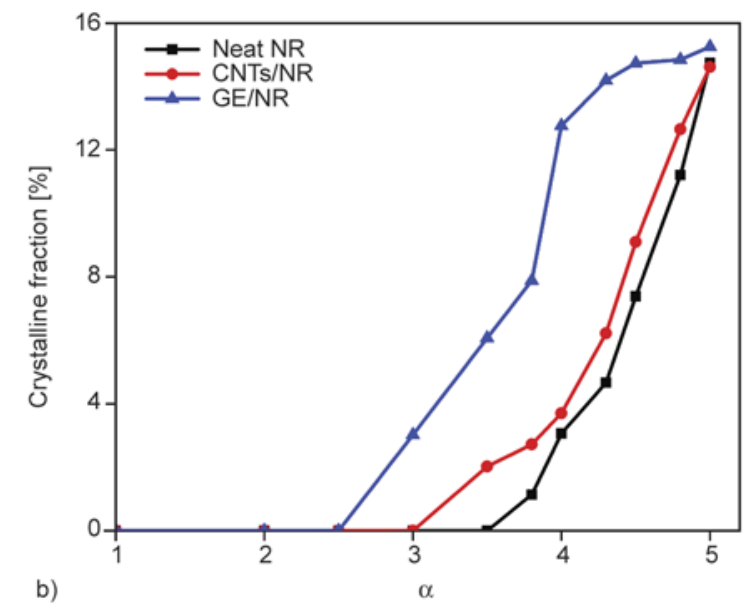

Figure 3. (a) Equatorial diffraction profiles taken from 2D WAXD patterns at selected extension ratios of GE/NR; (b) the crystallinity as a function of extension ratio for unfilled and filled NR composites

posites respectively. The crystallinity for three kinds of materials increase with respect to strain, and CNTs or GE filled NR composites exhibit higher crystallinity compared to unfilled NR at the same strain. GE/NR composite has the highest crystallinity among the three kinds of material. The crystallites formed along the chains during stretching can be regarded as quasi-fillers or alternatively as additional crosslinks in the rubber network, which plays a crucial role in enhancing mechanical properties of NR.
The onset extension ratio of crystallization $\left(\alpha^{0}\right)$ was obtained by the interception of the linear regression lines derived in the plot of crystallinity against strain. The calculated $\alpha^{0}$ value for the unfilled NR, $\mathrm{CNTs} / \mathrm{NR}$ and GE/NR are 3.5, 3.0 and 2.5 respectively. It is clear that SIC occurs at a lower $\alpha^{0}$ value in the filled NR samples, and GE/NR exhibits the lowest $\alpha^{0}$ value among the three samples. The low $\alpha^{0}$ value in GE/NR is thought to correlate with the high strain amplification effect of GE on NR matrix. The 
Table 3. Onset extension ratios of crystallization for unfilled NR and filled NR

\begin{tabular}{|l|c|c|c|}
\hline \multicolumn{1}{|c|}{ Samples } & $\boldsymbol{\alpha}^{\mathbf{0}}$ & $\boldsymbol{\alpha}_{\mathbf{r}}^{\mathbf{0}}(\boldsymbol{\varphi})$ & $\boldsymbol{\alpha}_{\mathbf{r}}^{\mathbf{0}}(\mathbf{b})$ \\
\hline NR & 3.5 & 3.5 & 3.5 \\
\hline CNTs/NR & 3.0 & 3.0 & 3.3 \\
\hline GE/NR & 2.5 & 2.5 & 3.2 \\
\hline
\end{tabular}

$\alpha^{0}$ : the experimental values, $\alpha^{0}(\varphi)$ : the corrected values considering the filler volume fraction, and $\alpha^{0}(b)$ : the corrected values considering the volume fraction of the fillers plus bound rubber.

effective strain of rubber portion in GE/NR is higher than the nominal macroscopic one by assuming GE un-deformable.

The relationship between the effective extension ratio and volume fraction $(\varphi)$ of the filler is formulated as Equation (6) [33]:

$\alpha_{\mathrm{r}}^{0}(\varphi)=\frac{\alpha^{0}-\varphi}{1-\varphi}$

The $\alpha^{0}$ values are converted in terms of Equation (6), and listed in Table 3. Because of the low filler content, the corrected value $\alpha_{\mathrm{r}}^{0}(\varphi)$ does not change compared with the experimental values $\left(\alpha^{0}\right)$. In filled rubber, the bound rubber layer around un-deformable filler particles is regarded as immobile, because of the intense physical and chemical interactions. The variation of the strain amplification effect in GE/NR and $\mathrm{CNTs} / \mathrm{NR}$ composites were interpreted in terms of different bound rubber contents in the two composites. On the basis of Leblanc and Hardy's methods [30], the measured bound rubber contents for CNTs/NR and GE/NR samples are 11.4 and 30.3\% (Table 2), respectively. The corrected onset strain $\alpha_{\mathrm{r}}^{0}(b)$ considering the filler plus bound rubber volume fraction are shown in Table 3. The corrected $\alpha_{\mathrm{r}}^{0}(b)$ values for filled NR are closer to the uncorrected values $\alpha^{0}$, but still much smaller than the onset

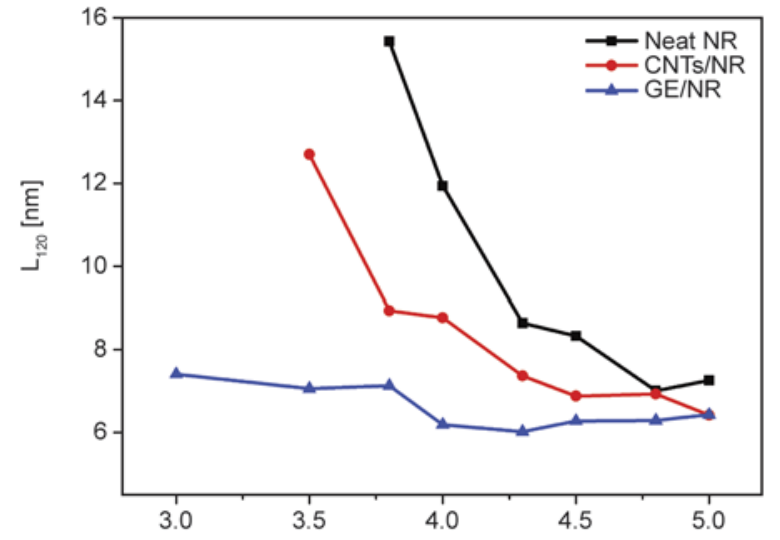

a)

$\alpha$ strain of unfilled NR. The addition of filler can dominate the rubber network structure and strain amplification effect. Also the GE or CNTs particles can act as nucleation centers of the NR crystallites. Raul et al. [34] thought that the addition of GE or CNTs makes the chains overstrained, and the crystallization was accelerated around particles.

\subsection{Lateral crystallite size}

Crystallite size is an important parameter in characterizing the crystallization behavior of rubber. The widths of peaks $I_{\text {eq }}(200)$ and $I_{\text {eq }}(120)$ were estimated and width $(w)$ value was converted into the halfwidth $\beta_{\text {hkl }}$ (represented by radian) according to the procedure described by Tosaka et al. [35]. The crystallite size was estimated using the Scherrer equation (Equation (7)):

$L_{\mathrm{hkl}}=\frac{k \lambda}{\beta_{\mathrm{hkl}} \cos \theta}$

where $L_{\mathrm{hkl}}$ is the crystallite size in the direction perpendicular to the $(h k l)$ plane which is parallel to the drawing direction, $\lambda$ is the wavelength, and $\theta$ is the Bragg angle (half of the scattering angle). The Scherrer factor $k$ is 0.89 when $\beta_{\text {hkl }}$ is the half width of the $(h k l)$ reflection in the radial direction [36]. Figure 4 shows the variations of the $L_{200}$ and $L_{120}$ crystallite sizes during stretching. Both $I_{\mathrm{eq}}(200)$ and $I_{\text {eq }}(120)$ decrease with strain, this is because the mean distance between the stretched chains acting as precursor of crystallite decreases, resulting in a decrease in the average crystallite size [36]. Since both GE and CNTs can act as the nuclei to induce the formation of crystallites during stretching, the filled NR samples show smaller crystallite sizes compared to the unfilled NR at the same strain. GE/NR

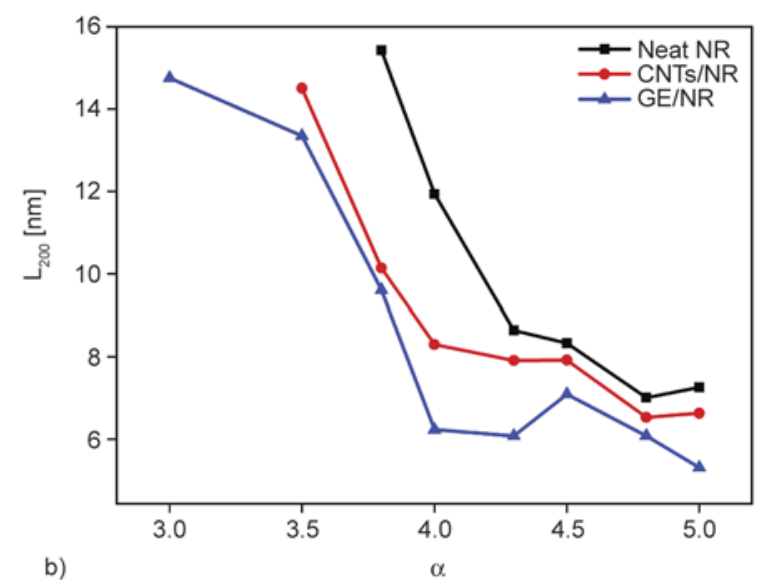

b)

Figure 4. Variation of lateral crystallite size: (a) $L_{120}$ and (b) $L_{200}$ during stretching 
has the smallest crystallite size among the three samples, because of the stronger interaction between GE and rubber.

\subsection{Entanglement-bound rubber tube (EBT) model}

EBT model was used to analyze the rubber network microstructure, to gain insight into the reinforcing effect of GE or CNTs on NR materials on molecular level. In EBT model, the packing effect arising from the inability of molecular chains to pass though their neighborhood imparts topological constraint acting on the entire network chains, and restricts the chains from fluctuating within small length scale. The EBT model allows an appropriate separation of crosslinks and constraint contribution to the stressstrain behavior, and enables the determination of crosslink density.

Based on the EBT model, the elastic free energy $w_{\mathrm{f}}$ that considers the finite extensibility of the chain together with the tube constraint can be expressed by two elastic moduli $G_{\mathrm{c}}$ and $G_{\mathrm{e}}$, which are closely related to the constants $C_{1}$ and $C_{2}$ in the MooneyRivlin equation [37]. The nominal stress $\sigma$ is obtained by differentiation $\sigma=\partial w_{\mathrm{f}} / \partial \alpha$ [38]. Based on the model, the constitutive equation of an incompressible sample can be expressed as Equations (8) and (9):

$\sigma^{*}=\frac{\sigma}{\alpha-\alpha^{-2}}=G_{\mathrm{c}}+G_{\mathrm{e}} \cdot f(\alpha)$

$f(\alpha)=\frac{2\left(\alpha^{\beta / 2}-\alpha^{-\beta}\right)}{\beta\left(\alpha^{2}-\alpha^{-1}\right)}, f(\alpha=1)=1$

where $\sigma^{*}$ is the reduced stress. $\sigma$ is the nominal stress. $\alpha$ is the extension ratio. $G_{\mathrm{c}}$ is the elastic modulus resulting from the crosslink constraint, and $G_{\mathrm{e}}$ is related to the topological tube-like constraint. $\beta$ is an empirical constant which describes the relation between the deformed tube in the stretched state and an undeformed tube corresponding to the equilibrium state, $\beta$ is generally taken as 1 [25]. $G_{\mathrm{e}}$ correlated with the tube lateral dimension within bulk rubber can be calculated by Equation (10):

$G_{\mathrm{e}}=\frac{v_{\mathrm{s}} l_{\mathrm{s}}^{2} k_{\mathrm{B}} T}{4 \sqrt{6} d_{0}^{2}}$

where $v_{\mathrm{s}}$ is the density of the statistical segments taken as $3.85 \mathrm{~nm}^{-3}$ [26], $l_{\mathrm{S}}$ is the average length of Kuhn's statistical segment $\left(0.88 \mathrm{~nm}\right.$ for NR). $k_{\mathrm{B}}$ is the Boltzmann constant. The lateral tube dimension $\left(d_{0}\right)$

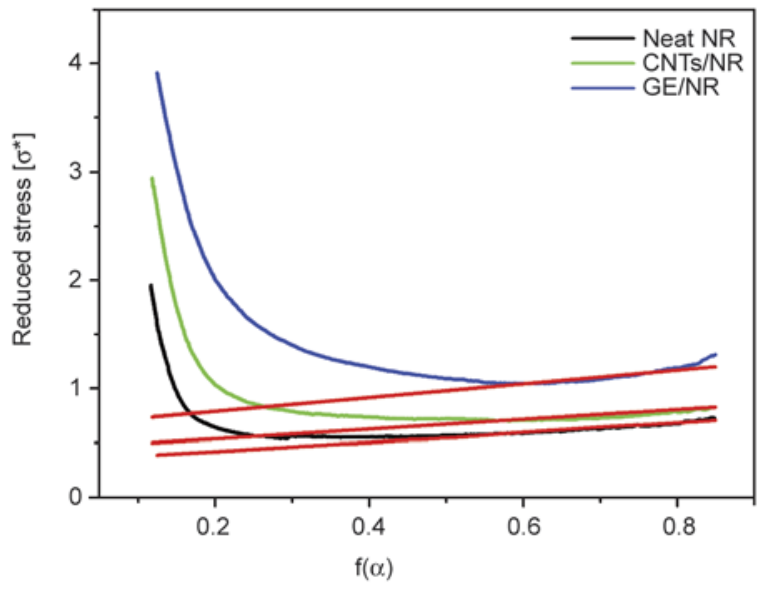

Figure 5. Mooney-Rivlin plots of the unfilled NR and NR composites. The red lines were obtained by linear fitting for the Mooney-Rivlin plots in the region of intermediate deformation

with a relationship with the mean number of statistical segments between successive entanglements $\left(n_{\mathrm{e}}\right)$ is expressed as Equation (11):

$d_{0}=l_{\mathrm{s}} \sqrt{n_{\mathrm{e}}}$

According to the Equation (8) and (9), the stressstrain curves in Figure 1 are converted into the form of a Mooney-Rivlin plot, $\sigma^{*}$ vs. $f(\alpha)$, as shown in Figure 5. Considering that EBT model is not fulfilled at large strains on the basis of molecular statistical theory [39], our analysis is limited in the intermediate deformation region. The parameters $G_{\mathrm{e}}$ (related to the topological tube like constraint) and $G_{\mathrm{c}}$ (contributed by the chemical crosslink) in Equation (8) can be determined from the slope and $y$-axis interception of the linear part of intermediate deformation, as shown in the Mooney plot $\sigma^{*}$ vs. $f(\alpha)$ (Figure 5), and the results are shown in Table 4. Given the fact that stress-strain behaviors of NR composite show strong dependence on both chemical crosslink and topological constraints contributions, the initial decrease in stress with increasing strain $\alpha$ can be ascribed to the slippage of entanglement [25]. At high strains, however, Mooney-Rivlin curves exhibit a noticeable upturn related to the rapidly increase of stress, which can be attributed to the strain induced crystallization [40] and the finite extensibility of chains [39]. As shown in Table 4, with the addition of filler, both network parameters $G_{\mathrm{c}}$ and $G_{\mathrm{e}}$ increase. Also it can be noted that the $G_{\mathrm{c}}$ and $G_{\mathrm{e}}$ values for GE/NR composite are higher than those for CNTs/NR composite. As shown in Figure 5, GE/NR composite 

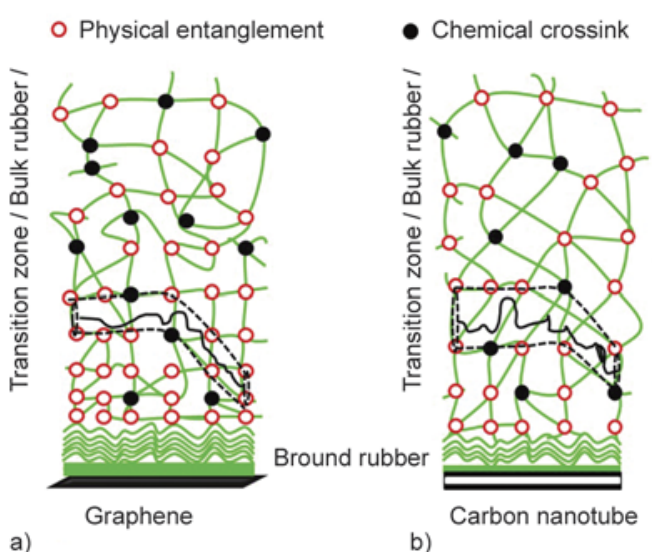

b)

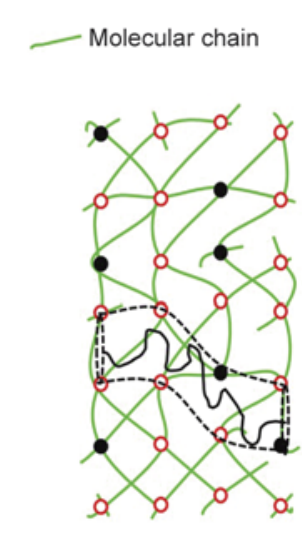

c)

Figure 6. Schematic diagram of the entanglement bound rubber tube model, (a) GE/NR (b) CNTs/NR (c) NR

shows an upturn at a smaller strain than $\mathrm{CNTs} / \mathrm{NR}$ composites, indicating that GE is more helpful for strain induced crystallization.

The average molecular mass of the network chains $\left(M_{\mathrm{c}}\right)$ is related to $G_{\mathrm{c}}$ according to the equation of $M_{\mathrm{c}}=$ $\rho_{\mathrm{p}} R T / G_{\mathrm{c}}, \rho_{\mathrm{p}}$ is the polymer density, $R$ is the gas constant. The decrease of $M_{\mathrm{c}}$ with increasing GE content is equal to an increase in the formation of polymer-filler couples and short bridging chains between filler particles which enlarges the extension of the filled network or the highly bounded clusters of filler particles [26]. Because of the stronger interfacial interactions between GE and rubber, GE/NR composites exhibit more increase in the parameters $G_{\mathrm{c}}$ and $G_{\mathrm{e}}$ than $\mathrm{CNTs} / \mathrm{NR}$ composites. To understand the effect of fillers on the rubber chain network, EBT model is schematically represented in Figure 6. The EBT model assumed that a number of entanglements, which are believed to dominate the rubber property, are formed in the transition zones between the tightly adsorbed bound and bulk rubbers far from the filler surface phases [25]. The parameters $G_{\mathrm{c}}$ and $G_{\mathrm{e}}$ values shown in Table 4 increased due to the strong interaction between fillers and rubber matrix in the transition zones. With the addition of filler, the tube dimension $d_{0}$ decreases, also the mean number of Kuhn's statistic chain segments between two successive entanglements $\left(n_{\mathrm{e}}\right)$, as well as the average molecular mass of the chain $\left(M_{\mathrm{c}}\right)$ decrease. Our results are similar with those for rubber network filled with clay reported by López-Manchado et al. [26].

The well dispersed GE or CNTs nanoparticles with high specific surface area have more chance, compared to the conventional filler with low specific surface, to interact with rubber matrix, and are capable of making majority of rubber confined even at a
Table 4. Network molecular parameters of unfilled and filled rubbers

\begin{tabular}{|l|c|c|c|c|c|}
\hline & $\begin{array}{c}\mathbf{G}_{\mathbf{c}}{ }^{{ }^{*} \mathbf{a}} \\
{[\mathbf{M P a}]}\end{array}$ & $\begin{array}{c}\mathbf{G}_{\mathbf{e}}{ }^{{ }^{\mathbf{b}}} \\
{[\mathbf{M P a}]}\end{array}$ & $\begin{array}{c}\mathbf{d}_{\mathbf{0}} \\
{[\mathbf{n m}]}\end{array}$ & $\mathbf{n}_{\mathbf{e}}$ & $\mathbf{M}_{\mathbf{c}}$ \\
\hline NR & 0.34 & 0.43 & 1.71 & 3.76 & 5389 \\
\hline CNTs/NR & 0.44 & 0.45 & 1.67 & 3.59 & 4164 \\
\hline GE/NR & 0.66 & 0.63 & 1.41 & 2.57 & 2776 \\
\hline
\end{tabular}

lower content. TEM and SEM micrographs confirm the homogeneous dispersion of GE or CNTs in NR matrix in our previous report $[14,41]$. So, as corroborated by molecular network parameters, the addition of GE or CNT reduces the tube dimensions, by the presence of adjacent chains, which restricts the movement of the rubber chains to a lower range. The chain is nanoscopically confined, forming a highly ordered and entangled structure due to the intense filler-rubber interactions, which limits the lateral fluctuation of the chain to a lower volume and reduces the number of possible chain configuration associated to the chain entropy [26]. In addition, the tube dimension for the GE/NR systems is smaller than that for CNTs/NR. This can be attributed to a more significant contribution of the entanglements in the transition zone due to the stronger filler-rubber interaction in the GE/NR composite. The lower $Q_{\mathrm{f}} / Q_{\mathrm{g}}$ value and higher bound rubber content of GE/NR shown in Table 2, confirm the strong interaction between the GE and NR matrix.

It is known that strain induced crystallization of NR results from the entropy drop during stretching, and this allows a thermodynamic description for SIC. The melting temperature $\left(T_{\mathrm{m}}\right)$ of NR increases during stretching. When $T_{\mathrm{m}}$ exceeds the room temperature, NR molecular chains are overstrained and get into a supercooled state, and the onset of crystallization take place, as shown in Equation (12) [42]: 
$\frac{1}{T_{\mathrm{m}, \mathrm{s}}}=\frac{1}{T_{\mathrm{m}, 0}}+\frac{\Delta S_{\mathrm{def}}}{\Delta H}$

where $T_{\mathrm{m}, \mathrm{s}}$ is the melting temperature in the stretched state, $T_{\mathrm{m}, 0}$ the melting temperature in the unstretched state, $\Delta H$ the melting enthalpy, and $\Delta S_{\text {def }}$ the difference of the entropy between the stretched and unstretched states. The onset of SIC of NR compos-

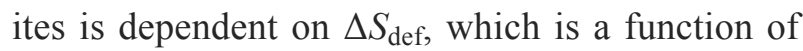
strain.

The entropy change of the filled rubber is consisted of two parts: (1) the entropy reduction caused by the addition of filler $\left(\Delta S_{\mathrm{f}}\right)$, which was attributed to the interactions between rubber chains and the filler; (2) deformation entropy $\left(\Delta S_{\mathrm{def}}\right)$ during stretching, as shown in Figure 7. $\Delta S_{\mathrm{f}}$ of CNTs/NR and GE/NR composites are expressed as $\Delta S_{1}$ and $\Delta S_{3}$, respectively. $\Delta S_{\text {def }}$ of CNTs/NR and GE/NR composites are expressed as $\Delta S_{2}$ and $\Delta S_{4}$, respectively. On the assumption that the total entropy change required for the onset of SIC in the filled and unfilled rubber is the same, i.e. $\Delta S_{0} \approx \Delta S_{1}+\Delta S_{2} \approx \Delta S_{3}+\Delta S_{4}$, as $\Delta S_{\mathrm{f}}$ of GE/NR composites is higher than that of CNTs/NR, i.e. $\Delta S_{3}>\Delta S_{1}$ due to a more ordered structure for GE/NR composites, $\Delta$ Sdef of GE/NR composites should be lower than that of CNTs/NR, i.e. $\Delta S_{4}<\Delta S_{2}$, which means the onset of strain-induced crystallization of GE/NR composites appeared at a lower strain.

\section{Conclusions}

Natural rubber composites containing two kinds of fillers, CNTs and graphene were prepared by an ultrasonically-assisted latex mixing process. A significant improvement in tensile strength was achieved for both graphene and CNTs filled NR, while graphene has a better reinforcing effect. The role of CNTs and graphene on strain-induced crystallization of NR was investigated by means of synchrotron WAXD. The addition of graphene in NR results in faster strain-induced crystallization rate and a higher crystallinity compared to CNTs, which contributes to a better reinforcing effect. The NR molecular network parameters of graphene/NR and CNTs/NR composites were determined by applying the EBT model in combination with Mooney-Rivlin plots. Due to the larger specific surface area, Graphene has more chance to interact with rubber than CNTs, resulting in the decreased tube dimension, and sequentially the reduction of configurational entropy of rubber molecular chains. Graphene/NR composites have lower deformation entropy during stretching than CNTs/NR composites.

\section{Acknowledgements}

This work is supported by National Natural Science Foundation of China (NNSFC 51433006) and the major project of Chinese Ministry of Education (313036). Professor Liangbin Li of National Synchrotron Radiation Laboratory (NSRL) in University of Science and Technology of China are also greatly appreciated for the help in Synchrotron WAXD experiments.

\section{References}

[1] Toki S., Sics I., Ran S., Liu L., Hsiao B. S., Murakami S., Senoo K., Kohjiya S.: New insights into structural development in natural rubber during uniaxial deformation by in-situ synchrotron X-ray diffraction. Macromolecules, 35, 6578-6584 (2002).

DOI: $10.1021 / \mathrm{ma} 0205921$

[2] Trabelsi S., Albouy P-A., Rault J.: Stress-induced crystallization around a crack tip in natural rubber. Macromolecules, 35, 10054-10061 (2002).

DOI: $10.1021 / \mathrm{ma} 021106 \mathrm{c}$

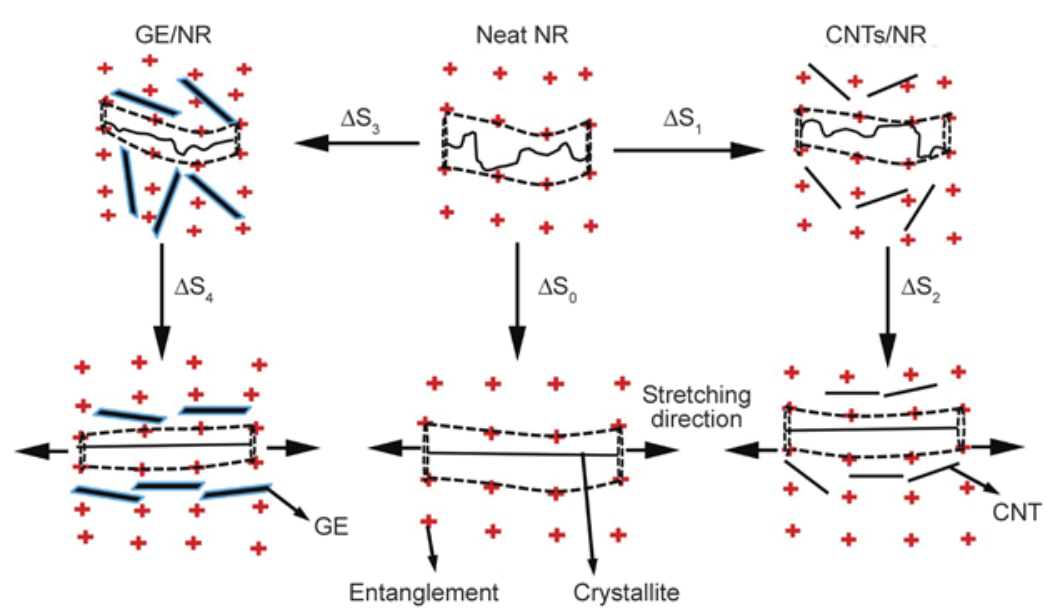

Figure 7. Schematic representation of structural evolution and entropy change of filled and unfilled rubber during stretching 
[3] Toki S., Hsiao B. S.: Nature of strain-induced structures in natural and synthetic rubbers under stretching. Macromolecules, 36, 5915-5917 (2003).

DOI: $10.1021 / \mathrm{ma} 034729 \mathrm{e}$

[4] Ikeda Y., Yasuda Y., Hijikata K., Tosaka M., Kohjiya S.: Comparative study on strain-induced crystallization behavior of peroxide cross-linked and sulfur crosslinked natural rubber. Macromolecules, 41, 5876-5884 (2008).

DOI: $10.1021 / \mathrm{ma} 800144 \mathrm{u}$

[5] Sun X., Sun H., Li H., Peng H.: Developing polymer composite materials: Carbon nanotubes or graphene? Advanced Materials, 25, 5153-5176 (2013).

DOI: $10.1002 /$ adma.201301926

[6] Kim H., Abdala A. A., Macosko C. W.: Graphene/polymer nanocomposites. Macromolecules, 43, 6515-6530 (2010).

DOI: $10.1021 / \mathrm{ma100572e}$

[7] Treacy M. M. J., Ebbesen T. W., Gibson J. M.: Exceptionally high Young's modulus observed for individual carbon nanotubes. Nature, 381, 678-680 (1996). DOI: $10.1038 / 381678 \mathrm{a} 0$

[8] Lee C., Wei X., Kysar J. W., Hone J.: Measurement of the elastic properties and intrinsic strength of monolayer graphene. Science, 321, 385-388 (2008). DOI: 10.1126/science.1157996

[9] Berber S., Kwon Y-K., Tománek D.: Unusually high thermal conductivity of carbon nanotubes. Physical Review Letters, 84, 4613-4616 (2000). DOI: 10.1103/PhysRevLett.84.4613

[10] Balandin A. A., Ghosh S., Bao W., Calizo I., Teweldebrhan D., Miao F., Lau C. N.: Superior thermal conductivity of single-layer graphene. Nano Letters, 8,902 907 (2008).

DOI: $10.1021 / \mathrm{n} 10731872$

[11] Ebbesen T. W., Lezec H. J., Hiura H., Bennett J. W., Ghaemi H. F., Thio T.: Electrical conductivity of individual carbon nanotubes. Nature, 382, 54-56 (1996). DOI: $10.1038 / 382054 \mathrm{a} 0$

[12] Geim A. K., Novoselov K. S.: The rise of graphene. Nature Materials, 6, 183-191 (2007). DOI: $10.1038 / \mathrm{nmat} 1849$

[13] Yan N., Xia H., Zhan Y., Fei G.: New insights into fatigue crack growth in graphene-filled natural rubber composites by microfocus hard-X-ray beamline radiation. Macromolecular Materials and Engineering, 298, 38-44 (2013).

DOI: $10.1002 /$ mame.201200044

[14] Zhan Y., Wu J., Xia H., Yan N., Fei G., Yuan G.: Dispersion and exfoliation of graphene in rubber by an ultrasonically-assisted latex mixing and in-situ reduction process. Macromolecular Materials and Engineering, 296, 590-602 (2011).

DOI: $\underline{10.1002 / \text { mame.201000358 }}$
[15] Zhan Y., Lavorgna M., Buonocore G., Xia H.: Enhancing electrical conductivity of rubber composites by constructing interconnected network of self-assembled graphene with latex mixing. Journal of Materials Chemistry, 22, 10464-10468 (2012).

DOI: $10.1039 / \mathrm{C} 2 J M 31293 \mathrm{~J}$

[16] Joly S., Garnaud G., Ollitrault R., Bokobza L., Mark J. E.: Organically modified layered silicates as reinforcing fillers for natural rubber. Chemistry of Materials, 14, 4202-4208 (2002). DOI: $10.1021 / \mathrm{cm} 020093 \mathrm{e}$

[17] Carretero-Gonzalez J., Verdejo R., Toki S., Hsiao B. S., Giannelis E. P., López-Manchado M. A.: Real-time crystallization of organoclay nanoparticle filled natural rubber under stretching. Macromolecules, 41, 22952298 (2008). DOI: $10.1021 / \mathrm{ma} 7028506$

[18] Li F., Yan N., Zhan Y., Fei G., Xia H.: Probing the reinforcing mechanism of graphene and graphene oxide in natural rubber. Journal of Applied Polymer Science, 129, 2342-2351 (2013).

DOI: $10.1002 / \mathrm{app} .38958$

[19] Song H. H., Kang M. K., Jeon H-J., Kwag G., Choi H.: Strain-induced crystallization and mechanical properties of carbon nanotubes reinforced natural rubber. in 'Technical Proceedings of the 2007 NSTI Nanotechnology Conference and Trade Show, Santa Clara, California' Vol 2, 164-165 (2007).

[20] Ozbas B., Toki S., Hsiao B. S., Chu B., Register R. A., Aksay I. A., Prud'homme R. K., Adamson D. H.: Straininduced crystallization and mechanical properties of functionalized graphene sheet-filled natural rubber. Journal of Polymer Science Part B: Polymer Physics, 50, 718-723 (2012). DOI: $10.1002 /$ polb. 23060

[21] Edwards S. F.: The statistical mechanics of polymers with excluded volume. Proceedings of the Physical Society, 85, 613-624 (1965). DOI: $10.1088 / 0370-1328 / 85 / 4 / 301$

[22] Edwards S. F.: The statistical mechanics of polymerized material. Proceedings of the Physical Society, 92, 9-16 (1967).

DOI: $10.1088 / 0370-1328 / 92 / 1 / 303$

[23] Leblanc J.: Insight into elastomer-filler interactions and their role in the processing behaviour of rubber compounds. Progress in Rubber and Plastics Technology, 10, 112-129 (1994).

[24] Funt J. M.: Dynamic testing and reinforcement of rubber. Rubber Chemistry and Technology, 61, 842-865 (1988).

DOI: $10.5254 / 1.3536222$

[25] Heinrich G., Vilgis T. A.: Contribution of entanglements to the mechanical properties of carbon blackfilled polymer networks. Macromolecules, 26, 11091119 (1993). DOI: $10.1021 / \mathrm{ma} 00057 \mathrm{a} 035$ 
[26] López-Manchado M. A., Valentín J. L., Carretero J., Barroso F., Arroyo M.: Rubber network in elastomer nanocomposites. European Polymer Journal, 43, 4143 4150 (2007).

DOI: $10.1016 /$ j.eurpolymj.2007.07.023

[27] Hummers W. S., Offeman R. E.: Preparation of graphitic oxide. Journal of the American Chemical Society, 80, 1339 (1958).

DOI: $10.1021 / \mathrm{ja} 01539 \mathrm{a} 017$

[28] Lorenz O., Parks C. R.: The crosslinking efficiency of some vulcanizing agents in natural rubber. Journal of Polymer Science, 50, 299-312 (1961).

DOI: $10.1002 /$ pol.1961.1205015404

[29] Ismail H., Nasaruddin M. N., Ishiaku U. S.: White rice husk ash filled natural rubber compounds: The effect of multifunctional additive and silane coupling agents. Polymer Testing, 18, 287-298 (1999).

DOI: 10.1016/S0142-9418(98)00030-0

[30] Leblanc J., Hardy P.: Evolution of bound rubber during the storage of uncured compounds. Kautschuk und Gummi, Kunststoffe, 44, 1119-1124 (1991).

[31] Treloar L. R. G.: The physics of rubber elasticity. Oxford University Press, Oxford (1975).

[32] Wu J., Schultz J. M., Yeh F., Hsiao B. S., Chu B.: Insitu simultaneous synchrotron small- and wide-angle $\mathrm{X}$-ray scattering measurement of poly(vinylidene fluoride) fibers under deformation. Macromolecules, 33, 1765-1777 (2000).

DOI: $10.1021 / \mathrm{ma} 990896 \mathrm{w}$

[33] Poompradub S., Tosaka M., Kohjiya S., Ikeda Y., Toki S., Sics I., Hsiao B. S.: Mechanism of strain-induced crystallization in filled and unfilled natural rubber vulcanizates. Journal of Applied Physics, 97, 103529/1103529/9 (2005).

DOI: $10.1063 / 1.1900927$

[34] Rault J., Marchal J., Judeinstein P., Albouy P. A.: Stress-induced crystallization and reinforcement in filled natural rubbers: ${ }^{2} \mathrm{H}$ NMR study. Macromolecules, 39, 8356-8368 (2006).

DOI: $10.1021 / \mathrm{ma} 0608424$
[35] Tosaka M., Murakami S., Poompradub S., Kohjiya S., Ikeda Y., Toki S., Sics I., Hsiao B. S.: Orientation and crystallization of natural rubber network as revealed by WAXD using synchrotron radiation. Macromolecules, 37, 3299-3309 (2004).

DOI: $10.1021 / \mathrm{ma} 0355608$

[36] Klug H. P., Alexander L. E.: X-ray diffraction procedures: For polycrystalline and amorphous materials. Wiley, New York (1974).

[37] Klüppel M., Schramm J.: A generalized tube model of rubber elasticity and stress softening of filler reinforced elastomer systems. Macromolecular Theory and Simulations, 9, 742-754 (2000).

DOI: 10.1002/1521-3919(20001201)9:9<742::AIDMATS742>3.0.CO;2-4

[38] Edwards S. F.: The theory of rubber elasticity. British Polymer Journal, 9, 140-143 (1977).

DOI: $10.1002 /$ pi.4980090209

[39] Wang M. C., Guth E.: Statistical theory of networks of non-Gaussian flexible chains. The Journal of Chemical Physics, 20, 1144-1157 (1952). DOI: $10.1063 / 1.1700682$

[40] Flory P. J.: Effects of molecular structure on physical properties of butyl rubber. Industrial and Engineering Chemistry, 38, 417-436 (1946). DOI: $10.1021 / \mathrm{ie} 50436 \mathrm{a} 023$

[41] Zhan Y. H., Liu G. Q., Xia H. S., Yan N.: Natural rubber/carbon black/carbon nanotubes composites prepared through ultrasonic assisted latex mixing process. Plastics, Rubber and Composites, 40, 32-39 (2011). DOI: $10.1179 / 174328911 X 12940139029284$

[42] Tosaka M.: A route for the thermodynamic description of strain-induced crystallization in sulfur-cured natural rubber. Macromolecules, 42, 6166-6174 (2009). DOI: $10.1021 / \mathrm{ma9} 00954 \mathrm{c}$ 